\title{
Near field characteristics of buoyant helium plumes
}

\author{
KUCHIMANCHI K BHARADWAJ ${ }^{1}$, DEBOPAM DAS ${ }^{1, *}$ and \\ PAVAN K SHARMA ${ }^{2}$
}

\author{
${ }^{1}$ Department of Aerospace Engineering, Indian Institute of Technology Kanpur, \\ Kanpur 208016, India \\ ${ }^{2}$ Reactor Safety Division, Bhabha Atomic Research Center, Mumbai 400085, India \\ e-mail: das@iitk.ac.in
}

MS received 16 May 2014; revised 18 December 2014; accepted 8 January 2015

\begin{abstract}
Puffing and entrainment characteristics of helium plumes emanating out into ambient air from a circular orifice are investigated in the present study. Velocity and density fields are measured across a diametric plane using Particle Image Velocimetry (PIV) and Planar Laser Induced Fluorescence (PLIF) respectively in phase resolved manner. Experiments are performed in Froude numbers range 0.2-0.4 and for Reynolds numbers 58-248. Puffing frequency measurements reveal that the plume puffing frequencies are insensitive to the plume exit conditions, since the instability is buoyancy driven. The frequencies obtained in the present case are in agreement with frequencies obtained by Cetegen \& Kasper (1996) for plumes originating from circular nozzles of various L/D ratios. Velocity and density measurements reveal that toroidal vortex formed during a puffing cycle entrains ambient air as it traverses downstream and this periodic engulfment governs the entrainment mechanism in pulsating plumes. The obtained velocity and density fields are used to calculate mass entrainment rates. It is revealed that though the flow is unsteady, the contribution of unsteady term in mass conservation to entrainment is negligible, and it becomes zero over a puff cycle. Finally, an empirical relation for variation of mass entrainment with height has been proposed, in which the non-dimensional mass entrainment is found to follow a power law with the non-dimensional height.
\end{abstract}

Keywords. Helium plume; puffing; PIV; PLIF; entrainment.

\section{Introduction}

Buoyancy induced fluid motion occurs frequently in nature and in many other engineering applications. The study of buoyant jets and plumes received considerable attention because of their relevance in understanding several flow configurations such as fuel leaks, pool fires, diffusion flames, hot jets and natural phenomena such as atmospheric thermals. The important parameter that classifies jets and plumes is inlet Richardson number (Yildirim \& Agrawal 2005) (ratio of

*For correspondence 
buoyancy to inertial forces, $R i_{i}=\frac{g D\left(\rho_{\infty}-\rho_{i}\right)}{\rho_{i} V^{2}}$, where $\rho_{\infty}$ and $\rho_{i}$ are ambient and inlet densities, $g, D$ and $V$ are gravity, inlet diameter and inlet velocity respectively), while the former is momentum dominated $\left(R i_{i} \ll 1\right)$, the latter is buoyancy dominated $\left(R i_{i}>1\right)$. The major difference in both classes of flows lies in the near field instabilities and flow transition. Of direct relevance to the present study is the pulsating instability observed in the near field of pool fires and buoyant diffusion flames, which is characterized by repetitive shedding of coherent vortices from the source at a defined frequency, commonly termed as puffing (Cetegen \& Ahmed 1993). These vortical structures arising from puffing control the entrainment of the ambient fluid in the near field. In fires, this entrainment controls the oxygen distribution in the near field, which affects the combustion. Puffing is also observed in low density gas plumes when the ratio of inlet to ambient densities (density ratio) $S<0.6$ (Cetegen 1997a). Though the dependence of puffing frequency on various flow parameters in reacting and non-reacting plumes is different, the flow field and vortex dynamics associated with both these classes of flows are similar. Hence, plumes generated using helium and helium-air mixtures, hot gases were used to understand the flow dynamics associated with complex flows such as flames and fires.

Cetegen and his co-authors pioneered the research in reacting and non-reacting buoyant plumes. In plumes of helium-air mixtures, they had witnessed the unsteady flow in the form of vortex like structures shedding from the nozzle exit. In planar (Cetegen et al 1998) and circular plumes (Cetegen \& Kasper 1996), they found that Strouhal number ( $S t=\frac{f D}{V}$, where $f$ is puffing frequency) scales with Richardson number $\left(R i_{\infty}=\frac{g D\left(\rho_{\infty}-\rho_{p}\right)}{\rho_{\infty} V^{2}}\right)$ by a power law. Cetegen (1997a) studied the characteristics of this instability for circular plumes emanating from nozzles of different $L / D$ ratios ( $L$-Length) and mapped the regions of instability on to $R e-S$ ( $R e=\frac{\rho_{p} V D}{\mu_{p}}$ and $S=\frac{\rho_{p}}{\rho_{\infty}}$, where $\rho_{p}, \mu_{p}$ are plume density and viscosity respectively) plane. By temporally visualizing a starting plume from laminar to pulsating state, he termed this instability to be convective, which was later contradicted by Soteriou et al (2002). They performed Lagrangian simulations on planar buoyant plumes and concluded this instability to be absolute. Cetegen (1997b) performed velocity measurements using DPIV for a plume of $R i_{i}=283$ and found that the vortex forms at point of zero stream-wise acceleration. Jiang \& Luo (2000) performed DNS in axisymmetric thermal plumes and investigated mechanism leading to puffing, based on vorticity transport. Quantitative measurements of velocity and density fields have been largely confined to large scale turbulent helium plumes of $D=1 \mathrm{~m}$ (DesJardin et al 2004; O'Hern et al 2005). These studies are intended to develop turbulence models for fire flows, based on the velocity and density data obtained from simultaneous PIV and PLIF measurements in large scale helium plumes. Maragkos et al (2013) performed Large Eddy Simulations (LES) to study the vortex dynamics associated with $D=1 \mathrm{~m}$ plume.

Most of the studies pertaining to transitional buoyant plumes are confined to the flow visualization and frequency measurements. However, in such unsteady flows, simultaneous measurement of velocity and density fields with high temporal resolution is important to study the instability mechanism, vortex dynamics and entrainment characteristics of pulsating plumes. The present work is aimed to address some of the above issues by investigating puffing and entrainment characteristics of helium plumes emanating out of a circular orifice. Phase resolved velocity and density measurements in a diametric plane of the plume are carried out using PIV and PLIF and puffing frequencies are measured using hot-wire probe. A detailed description about the experimental set-up and measurements is given in Section 2. The non-dimensional groups governing the plume behaviour are Reynolds number $R e$, Froude number $F r$, Density ratio $S$ and Schmidt number $S c\left(R e=\frac{\rho_{p} V D}{\mu_{p}}, F r=\frac{V}{\sqrt{g D}}, S=\frac{\rho_{p}}{\rho_{\infty}}, S c=\frac{\mu_{p}}{\rho_{p} \alpha}\right.$ where $\mu_{p}$ and $\alpha$ are plume 
Table 1. Re-Fr parameter space for the present experiments (PIV and PLIF performed for highlighted cases only).

\begin{tabular}{lccccc}
\hline $\boldsymbol{D}(\mathbf{m m})$ & \multicolumn{5}{c}{$\boldsymbol{F r}$} \\
\cline { 2 - 6 } & $\mathbf{0 . 2 0 0}$ & $\mathbf{0 . 2 5 0}$ & $\mathbf{0 . 3 0 0}$ & $\mathbf{0 . 3 5 0}$ & $\mathbf{0 . 4 0 0}$ \\
\hline $\mathbf{5 0 . 0}$ & $\operatorname{Re}=58$ & 73 & 87 & 102 & 116 \\
$\mathbf{6 0 . 8}$ & 78 & 97 & 117 & 136 & 155 \\
$\mathbf{7 7 . 0}$ & 111 & 139 & 166 & - & - \\
$\mathbf{1 0 0 . 6}$ & 166 & 207 & 248 & - & - \\
\hline
\end{tabular}

viscosity and mass diffusivity respectively). Unlike previous investigations, which involved a simultaneous variation of these groups, the helium plumes in present study are investigated by varying $R e$ for a constant $\mathrm{Fr}$ as shown in table 1. Four orifices of different diameters are used and pre-calculated inlet mass flow rates are set such that $F r$ remains constant and $R e$ varies w.r.t. diameter.

\section{Experiments}

The schematic of experimental set-up is shown in figure 1 . The set-up primarily consists of a gas flow circuit, settling chamber and an orifice through which the plume ejects out. The gas flow circuit comprises of helium gas cylinder $(50 \mathrm{~L})$, Mass Flow Controllers (MFCs), and high pressure tubing. Helium gas enters the bottom of the settling chamber, passing through two MFCs (Alicat Scientific MFC-100SLPM, MFC-1000SLPM). Inlet mass flow rate is set using these two MFCs as per the parameters shown in table 1. In the settling chamber, three 40 mesh, flat stainless steel screens are placed normal to the flow as shown in figure 1. These screens are placed in order to breakdown the larger eddies formed at the entry of helium gas and to ensure a more uniform flow in the settling chamber. The top open end of the settling chamber is concealed using a $9 \mathrm{~mm}$ thick acrylic sheet, which has an opening at its center for flush mounting

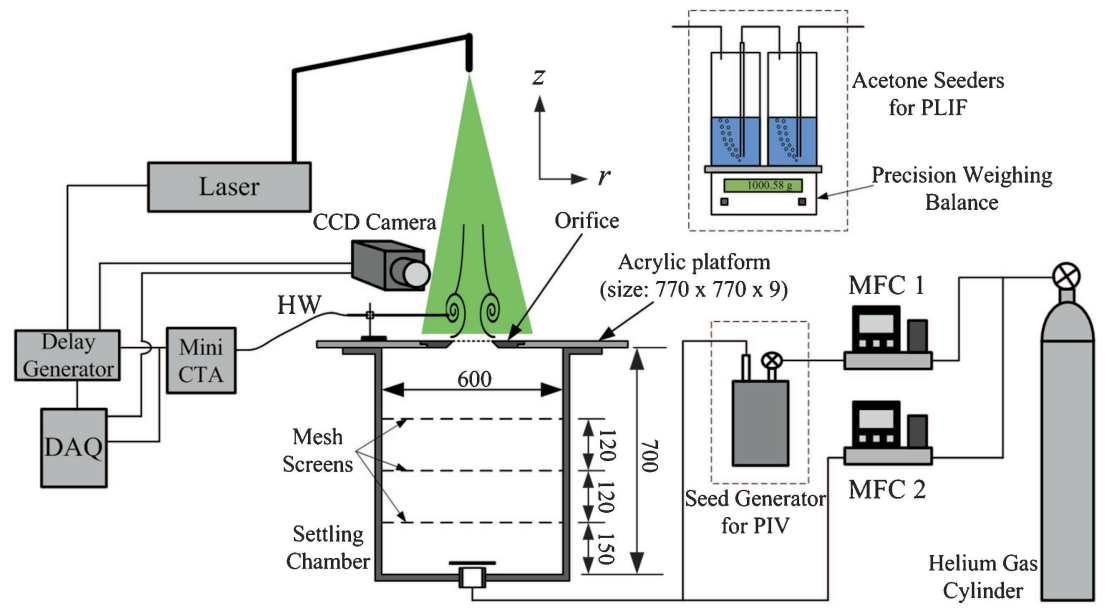

HW: Hot Wire probe MFC: Mass Flow Controller

All dimensions in $\mathrm{mm}$

Figure 1. Schematic of experimental set-up. 
the orifices as shown. Orifices of diameters 50, 60.8, 77 and $100.6 \mathrm{~mm}$ are fabricated using an acrylic sheet of $9 \mathrm{~mm}$ thickness. A 40 mesh, flat stainless steel screen is placed across the orifice exit. The main purpose of this screen is to prevent back flow of air, upstream of orifice exit and provide a uniform flow condition at the nozzle exit, which is otherwise impossible due to rapid buoyant acceleration of helium as it enters ambient air (Cetegen \& Kasper 1996). The plume is surrounded by a square shaped enclosure (size: $1250 \times 1250 \mathrm{~mm}$, height: $2400 \mathrm{~mm}$ from the ground) to prevent the plume from any room drafts.

In order to resolve velocity and density fields in a puffing cycle with high temporal resolution, PIV and PLIF measurements are carried out in phase resolved manner. This has been possible in the present work, as the flow near the plume exit is periodic. A hotwire probe is placed normal to stream-wise direction, at $(r, z)=(0.5 \mathrm{D}, 0.5 \mathrm{D})$ (axes shown in figure, origin at the center of the orifice exit) and its signal is fed to a digital delay generator. The laser and the camera are delayed with respect to the hotwire signal to resolve various phases in a puffing cycle. Simultaneously, this hotwire signal is acquired at $1000 \mathrm{~Hz}$ for $20 \mathrm{~s}$ using a NI 4472, 24-bit Data Acquisition (DAQ) card, and is used for calculating puffing frequency. The details of PIV and PLIF are given below.

\section{Velocity field measurements}

PIV is performed to measure the instantaneous 2-D velocity field. The flow field is illuminated using a double pulsed Nd-YAG laser (Quanta System, $10 \mathrm{~Hz}, 300 \mathrm{~mJ} / \mathrm{pulse}$ ). The light sheet is delivered to the test section using a light arm as shown. The light-sheet thickness achieved is typically about $1 \mathrm{~mm}$. The sheet is aligned to the centre line of the orifice and cuts the diametric plane. A 14-bit CCD camera (Pixelfly USB, $1392 \times 1040$ pixels resolution) is used for capturing the seed particle images. The camera is operated at a frame rate of $5 \mathrm{~Hz}$ in double frame mode. Voltage signal from a mini-CTA (Dantec Dynamics) is used for triggering the PIV acquisition system. The PIV acquisition system comprises of a 16 channel digital delay and pulse generator (Sequencer V801-BM, Hardsoft Microprocessor Systems), which synchronizes the laser and camera. Two economical seed generators (Jermy 2002), developed in-house, are used for seeding the flow field. The first one which is kept in the gas flow circuit as shown in the figure is used to seed the helium plume. Seeding concentration levels have been adjusted by varying the flow rate through MFC1 and by-passing the remaining flow through MFC2. Prior to each experiment, the air in the enclosure surrounding the plume has been seeded using the second seed generator, in order to capture the velocity field of ambient fluid entraining into the plume. High pressure air (from an air compressor) is used to generate seeding from the second seed generator. Edible soya-bean oil is used as working fluid in both the seed generators that generate oil particles of size $\approx 2-3 \mu \mathrm{m}$. Dynamic Studio software of Dantec Dynamics is used for analysing the captured particle images and obtain velocity fields. This analysis is carried out with interrogation window of size $32 \times 32$ pixels and an adaptive correlation with a $50 \%$ overlap.

\section{Density field measurements}

PLIF is performed to measure planar density/concentration field of the plume. Acetone vapour is used as a passive tracer for PLIF measurements. The experimental set-up used for PLIF is the same as that of used for PIV, apart from three modifications namely, (1) the output wavelength of the pulsed laser has been switched from $532 \mathrm{~nm}$ to $266 \mathrm{~nm}$ (90 mJ/pulse), (2) CCD camera replaced with an ICCD camera (DiCam Pro) and (3) seed generator replaced with Acetone seeders (refer figure 1). The helium plume is seeded with acetone vapour by passing helium gas through two aluminum chambers connected in series with MFC1, containing liquid acetone as 
shown. The two chambers are kept on a precision weighing balance and the amount of liquid acetone leaving the chambers per unit time (acetone concentration) is recorded during calibration and experiments. Acetone concentration is controlled by setting helium flow rate in MFC1 and bypassing excess helium gas through MFC2, to achieve desired concentration levels at plume exit.

Acetone fluorescence equation for weak excitation is given by Thurber (1999). For an isothermal, isobaric flow and for a fixed wavelength excitation, the fluorescence signal is directly proportional to the local acetone concentration and laser energy (Thurber 1999; Lozano et al 1992). Acetone vapour in the flow is excited using $266 \mathrm{~nm}$ wavelength and the emitted fluorescence is collected in the visible wavelength range 350-550 nm (Lozano et al 1992). The glass lens acts as a filter to block any UV light and prevents fluorescence signal contamination. Since the fluorescence signals are relatively weak, an ICCD has been used to achieve high S/N ratio. Prior to the experiments, the fluorescence emitted is calibrated for several known concentrations of acetone-air mixtures. An acrylic chamber filled with a known, uniform mixture of acetone vapour and air, is placed at the plume exit and illuminated using laser sheet. The fluorescence images, thus obtained by imaging different mixture concentrations, are used for calibration. These calibration images also aid in normalizing the PLIF images in order to correct for laser sheet spatial non-uniformities. The raw PLIF images obtained are corrected for laser sheet spatial variations, pulse-to-pulse variations (temporal), background light and dark noise by adopting the algorithm outlined in Clemens (2002) and Martin \& Garcia (2009). A MATLAB image processing based program has been developed in-house to process these raw fluorescence images into helium concentration fields, based on the algorithm. The attenuation of laser intensity due to acetone is negligible as very weak acetone concentrations have been used (mole fraction $<4 \%$ ). Once the concentration field is mapped throughout the image, the density is found from the linear transformation as given in Martin \& Garcia (2009).

\section{Results and discussion}

\subsection{Puffing frequency}

The pulsation frequency is determined by performing a Fast Fourier Transform (FFT) of the hotwire signal. A truncated portion of hot-wire signal and corresponding FFT for the case $R e=166$ and $F r=0.3$ are shown in figure 2(a) and 2(b) respectively. The hot-wire signal shows that the flow in the near field is periodic and its FFT shows a dominant frequency of $5.95 \mathrm{~Hz}$. Cetegen $\&$ Kasper (1996) experimentally studied the dependence of puffing frequency on various input parameters, using unsteady pressure measurements in near-field of circular buoyant plumes emanating from nozzles. They found that the Strouhal number $S t$ scales with Richardson number $R i_{\infty}$ by a relation $S t=0.8 R i_{\infty}^{0.38}$ in $R i_{\infty}$ range $1-100$. Figure 2(c) gives the variation of $S t$ with $R i_{\infty}$ for the present experiments. The $S t$ obtained for orifice generated plumes in the present work is in agreement with the scaling proposed by Cetegen \& Kasper (1996) for nozzle generated plumes. This implies that the flow instability is predominantly buoyancy dominated at the near field and change in shear has negligible effect on puffing phenomenon. This agreement also demonstrates the validity of the present experimental set-up and measurements.

Figure 3 shows the axial velocity variation with time, obtained from phase resolved PIV measurements, over the centerline ( $r=0$ ), at various locations along the axis (at $z=0.5 \mathrm{D}, 1 \mathrm{D}, 1.5 \mathrm{D}$ and 2D) for the same case in figure 2(a). It can be observed that the velocity field in the near-field is quite periodic with distinct frequency. Further away from the nozzle exit, the flow becomes complex and additional frequencies are also present along with the puffing frequency. It is noted 

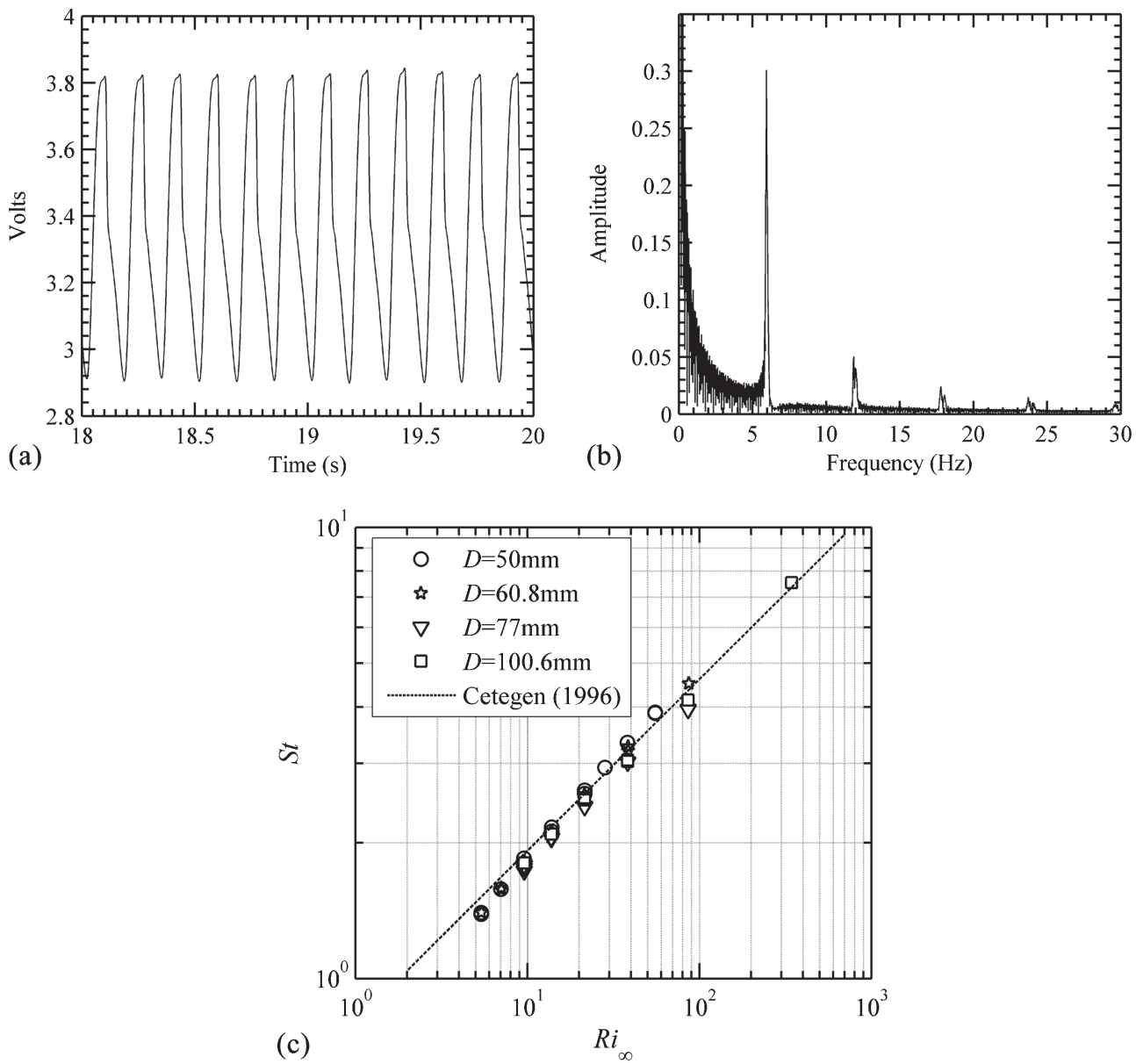

Figure 2. (a) Hot-wire signal for the case $R e=166$ and $F r=0.3$ (b) FFT of (a); and (c) variation of Strouhal number with Richardson number.

that velocities probed at any position in the flow field for the case shown in figure 3 yield the same dominant frequency, indicating a global absolute instability. Figure 4 shows the variation of $S t$ with $R e$ for various $F r$. It is observed that the $S t$ is relatively independent of $R e$ and depends only on $\mathrm{Fr}$, which reveals that the puffing instability mechanism is inviscid. This is consistent with the observations made by Soteriou et al (2002) in the case of planar buoyant plumes.

\subsection{Flow field during a puffing cycle}

Flow visualization, velocity, vorticity and density fields during five different phases of a typical puffing cycle i.e. $t=0.10,0.14,0.18,0.22$ and $0.26 \mathrm{~s}$ for the case discussed in figure 3 (time period for one puff cycle $t_{\mathrm{p}}=0.168 \mathrm{~s}$ ), are shown in figure 5 , to explain the flow field and vortex dynamics associated with a pulsating plume. In buoyant flows, vorticity is predominantly generated from baroclinic torque (misalignment between pressure and density gradients) and gravitational torque (misalignment between density gradient and gravity). Many numerical studies of buoyant plumes (Jiang \& Luo 2000; Soteriou et al 2002; DesJardin et al 2004; Maragkos et al 2013) have identified that gravitational torque (buoyancy) is the dominant term 

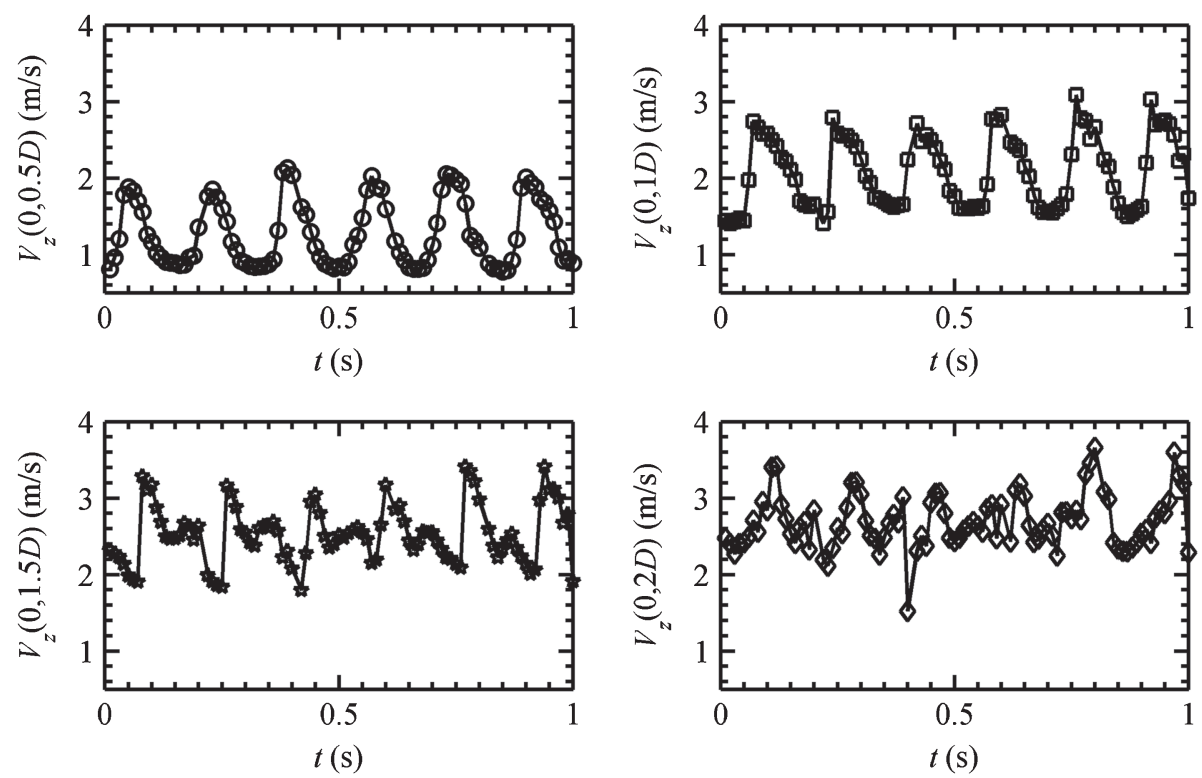

Figure 3. Axial velocity variation with time at various axial locations along the centerline for $R e=166$ and $F r=0.3$.

controlling the plume near-field dynamics. At time $t=0.10 \mathrm{~s}$, it can be observed that the misalignment of density gradient and gravity is maximum near the exit i.e. density gradient acting radially outward and gravity in the vertical direction. This leads to the vorticity generation and serves to initiate the base instability when viscous forces are less dominant. This instability leads to the creation of a coherent toroidal vortex, as shown at $t=0.14,0.18$ and $0.22 \mathrm{~s}$. The vortex grows rapidly under the influence of gravitational torque and baroclinic torques (generated due to pressure gradient generated by rapid acceleration of lighter fluid and vortex). The rapid buoyant acceleration of plume fluid along the centerline leads to the stretching of the toroidal vortex

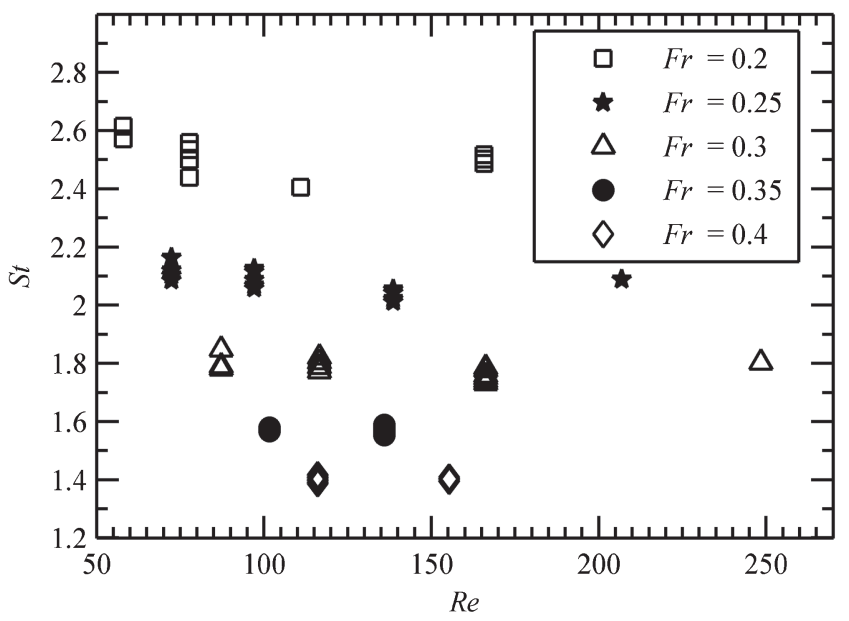

Figure 4. St variation with $R e$ for puffing plumes of various $F r$. 

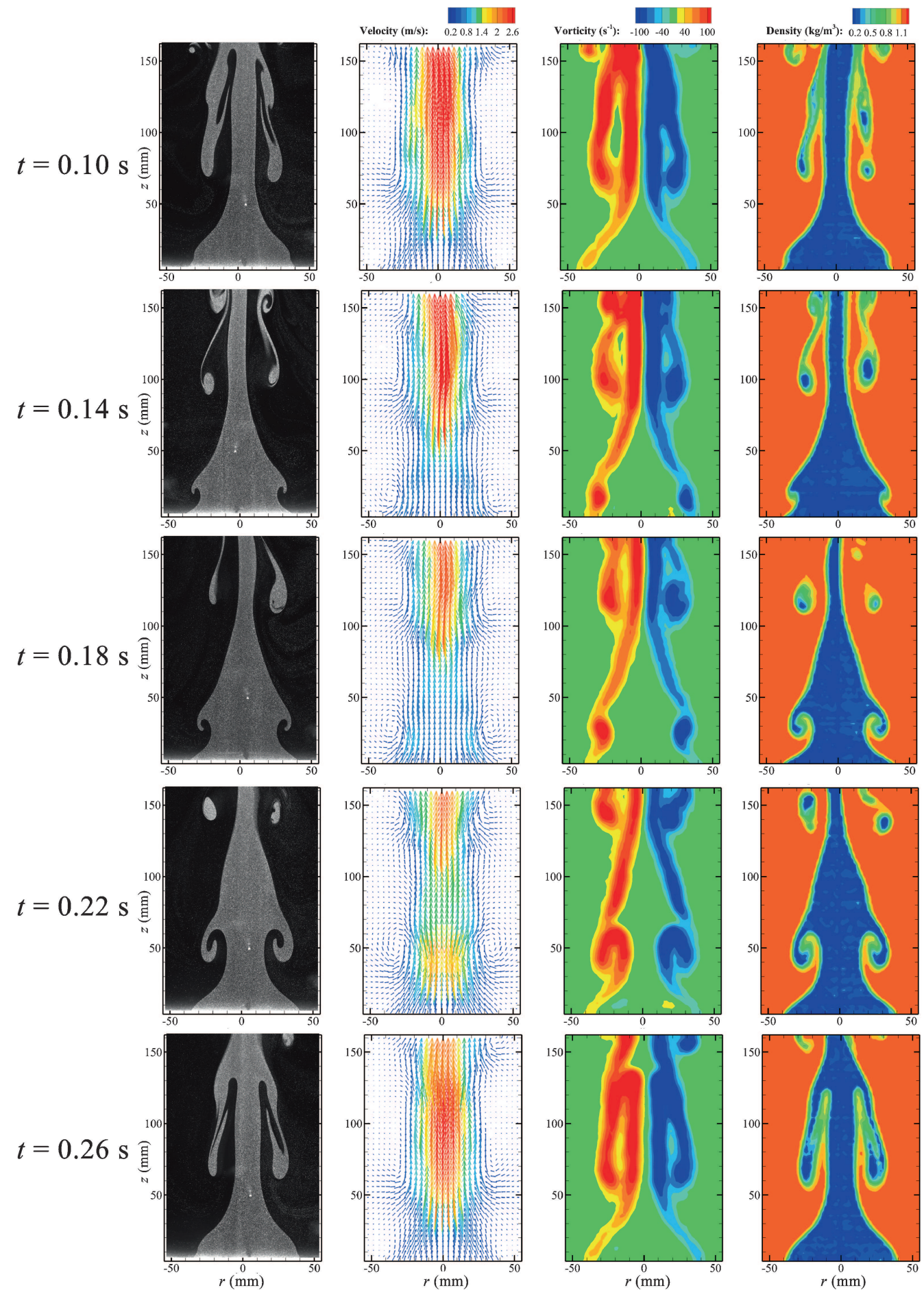

Figure 5. Flow field during different phases of a puffing cycle for $\operatorname{Re}=166$ and $F r=0.3$. 
$(t=0.26 \mathrm{~s})$, which is translating downstream at a relatively lower velocity while entraining ambient fluid. This phenomenon leads to secondary azimuthal instabilities which breaks the toroidal vortex and leads to rolling up of series of vortices as shown at $t=0.10$ and $0.14 \mathrm{~s}$. The centerline velocity reveals a stronger acceleration of the plume fluid along the centerline followed by deceleration at the position of the toroidal vortex at any time instant. The buoyant acceleration of plume fluid is accompanied by radial inflow of ambient fluid towards the plume centerline which is observed at all-time instants in a puff cycle. The velocity near the plume exit attains minimum, just before the start of a puffing cycle. This leads to the necking of the plume fluid from the previous puffing cycle $(t=0.26 \mathrm{~s})$ and accumulation of fluid near the plume base for next puffing cycle. It is observed that the centerline density remains equal to the inlet density for a longer axial distance, thus preserving the plume buoyancy.

Thus, from the flow field, it is inferred that the toroidal vortex engulfs ambient fluid during its growth, convection and break-up during a puffing cycle. This periodic engulfment governs the entrainment mechanism in pulsating buoyant plumes.

\subsection{Mass entrainment}

The velocity and density fields obtained are used to calculate mass entrainment rates. At any time $t$, an axisymmetric control volume (CV), as shown in figure 6 , is chosen to calculate instantaneous mass entrainment up to an axial distance $z=Z$, using mass conservation. The instantaneous mass entrainment thus obtained is integrated over one second to obtain mass entrainment rate. The equations used are as follows.

Mass entrainment at any instant $t$ up to an axial distance $z=Z$ is given by

$$
\dot{m}_{\text {ent }}(Z, t)=\underbrace{2 \pi \frac{\partial}{\partial t} \int_{0}^{Z} \int_{0}^{R_{C V}} \rho(r, z, t) r d r d z}_{\dot{m}_{\text {unsteady }}(Z, t)}+\underbrace{2 \pi \int_{0}^{R_{C V}} \rho(r, Z, t) V_{z}(r, Z, t) r d r}_{\dot{m}_{\text {out }}(Z, t)}-\dot{m}_{\text {in }}
$$

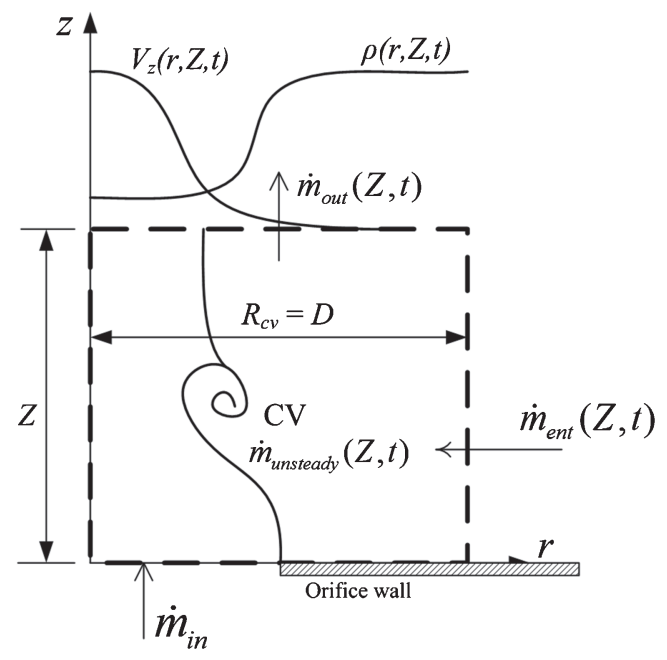

Figure 6. Schematic of control volume chosen at any time instant $t$ for calculating mass entrainment. 
Mass entrainment till an axial distance $z=Z$ in one second is given by

$$
\dot{m}_{\text {ent }}(Z)=\underbrace{\int_{0}^{1} \dot{m}_{\text {unsteady }}(Z, t) d t}_{\dot{m}_{\text {unsteady }}(Z)}+\underbrace{\int_{0}^{1} \dot{m}_{\text {out }}(Z, t) d t}_{\dot{m}_{\text {out }}(Z)}-\dot{m}_{\text {in }}
$$

Here, $\dot{m}_{i n}$ is the plume inlet mass flow rate in $\mathrm{kg} / \mathrm{s} . \dot{m}_{\text {unsteady }}(Z, t)$ is the rate of change of mass in the CV and $\dot{m}_{\text {out }}(Z, t)$ is mass flux at $z=Z$, at any time instant $t$ (refer figure 6). $\dot{m}_{\text {unsteady }}(Z)$ and $\dot{m}_{\text {out }}(Z)$ are the rate of change of mass in CV and mass flux (at $z=Z$ ) over one second.

Before calculating mass entrainment rate, the contribution of each term in Eqs. 1 and 2 is investigated to understand the entrainment phenomenon. Figure 7(a) compares $\dot{m}_{\text {unsteady }}(Z, t)$ and $\dot{m}_{\text {out }}(Z, t)$ for the case $R e=166 \mathrm{Fr}=0.3$ (same case used in figures 3 and 5) obtained at an axial distance $Z=50 \mathrm{~mm}$ from plume inlet. The highlighted markers in figure 7(a) correspond to the 5 time instants shown in figure 5 . It is observed that $\dot{m}_{\text {unsteady }}(Z, t)$ and $\dot{m}_{\text {out }}(Z, t)$ are out of phase by $180^{\circ}$ i.e. an increase in $\dot{m}_{\text {out }}(Z, t)$ leads to a decrease in $\dot{m}_{\text {unsteady }}(Z, t)$ and viceversa. Furthermore, over a puffing cycle, the $\dot{m}_{\text {unsteady }}(Z, t)$ is periodic about 0 and its frequency is equal to the puffing frequency. This shows that over a puffing cycle of time period $t_{p}, \int_{t}^{t+t_{p}} \dot{m}_{\text {unsteady }}(Z, t) \mathrm{dt}=0$. Also, the total variation of $\dot{m}_{\text {unsteady }}(Z, t)$ in one second ( $\dot{m}_{\text {unsteady }}(Z)$ ) is negligible when compared to $\dot{m}_{\text {out }}(Z)$. Figure 7(b) compares the variation of $\dot{m}_{\text {unsteady }}(Z)$ and $\dot{m}_{\text {out }}(Z)$ with axial distance $Z$ for $F r=0.3$ and $R e=166,117$ and 87. It can be observed that $\dot{m}_{\text {unsteady }}(Z)$ is negligible when compared to $\dot{m}_{\text {out }}(Z)$ over the axial distance and its magnitude $\approx 0$. However, it is to be noted that when considering instantaneous entrainment rate at any time $t$, the magnitude of $\dot{m}_{\text {unsteady }}(Z, t)$ is comparable to that of $\dot{m}_{\text {out }}(Z, t)$ and cannot be neglected. From the above analysis, it can be concluded that time averaged (mean) velocity and density fields would be sufficient to calculate the mass entrainment rates in pulsating buoyant plumes, since the contribution of unsteady term to mass entrainment rate is negligible.

Figure 8(a) shows the entrainment variation with axial distance $Z$ for $F r=0.3,0.25$ and 0.2 and for various $R e$ highlighted in table 1 . The mass entrainment rates $\left(\dot{m}_{\text {ent }}(Z)\right)$ calculated along
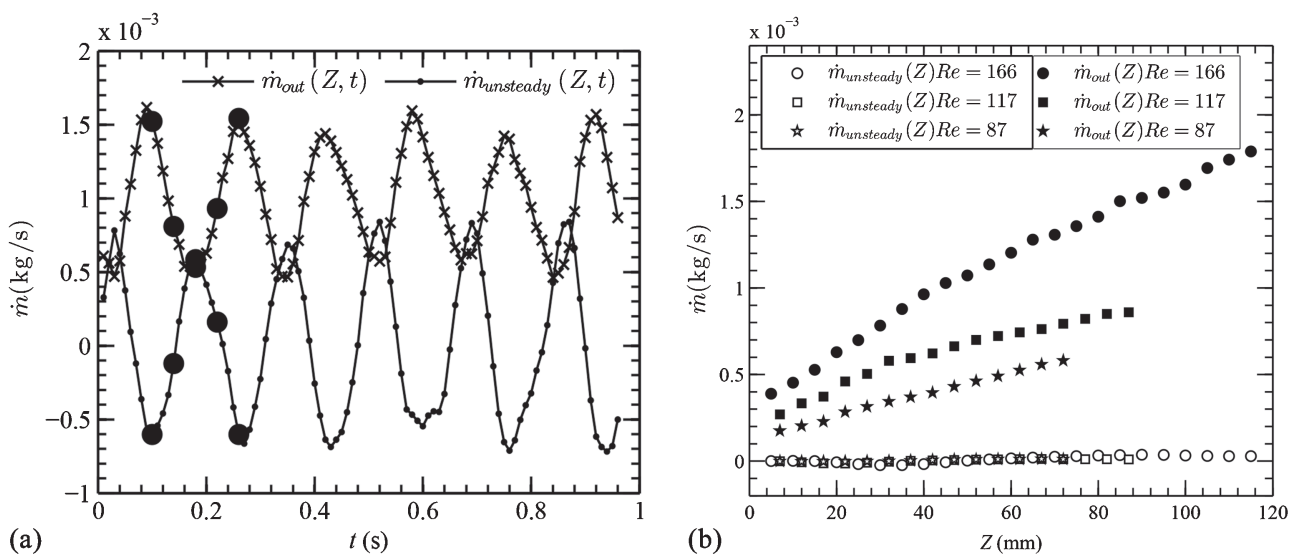

Figure 7. (a) Variation of $\dot{m}_{\text {unsteady }}(Z, t)$ and $\dot{m}_{\text {out }}(Z, t)$ with time, calculated at $Z=50 \mathrm{~mm}$ for the case shown in figure 5. (b)Variation of $\dot{m}_{\text {unsteady }}(Z)$ and $\dot{m}_{\text {out }}(Z)$ with axial distance $Z$ for $F r=0.3$ and $R e=166$, 117 and 87. 

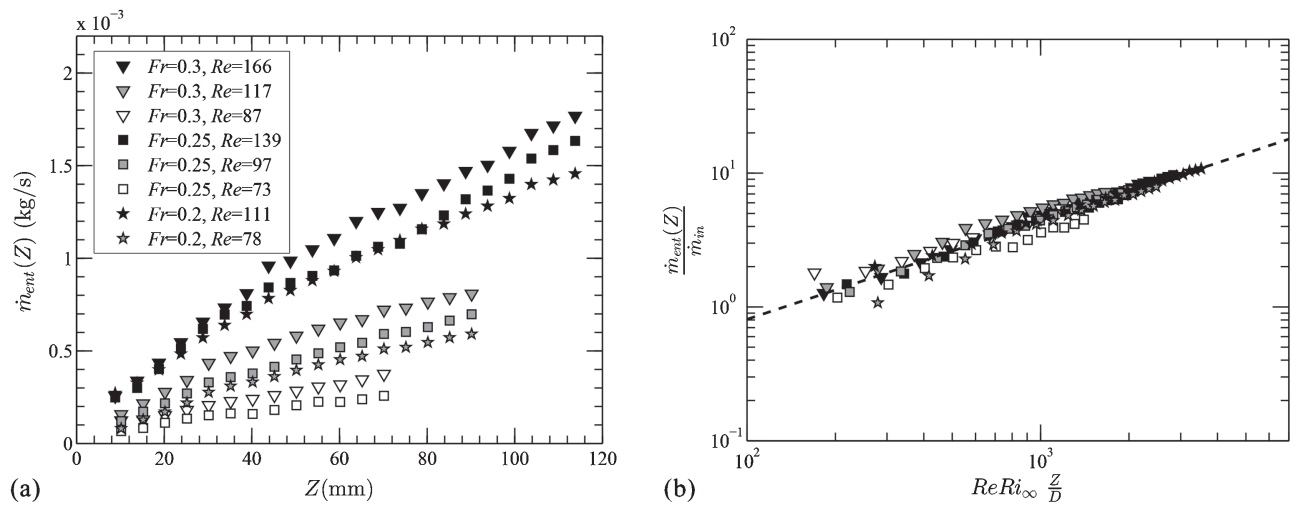

Figure 8. (a) Mass entrainment variation with axial distance. (b) Mass entrainment scaling.

axial distance $(Z)$ are scaled with various governing parameters to obtain an empirical relation for variation of $\dot{m}_{e n t}(Z)$ with $Z$ and eliminate its dependence on various inlet parameters. The data converged reasonably well when $\dot{m}_{\text {ent }}(Z)$ is scaled with $\dot{m}_{i n}$ and $Z$ with $\frac{D}{\operatorname{Re} R i_{\infty}}$; and the data is best fitted $\left(r^{2}=0.95\right)$ using a power law as shown in figure $8(b)$. Thus, in the present experiments, the mass entrainment rate is found to correlate with axial distance as

$$
\frac{\dot{m}_{\text {ent }}(Z)}{\dot{m}_{\text {in }}}=0.028\left(\operatorname{Re} \operatorname{Ri}_{\infty} \frac{Z}{D}\right)^{0.73}
$$

It is expected that the scaling law proposed also accounts for plumes of different densities due to the presence of $S$ in scaling i.e. $R i_{\infty}=\frac{1-S}{F r^{2}}$.

\section{Conclusions}

Puffing and entrainment characteristics of helium plumes emanating from circular orifices are investigated in the present study. Velocity and density measurements are performed in a phase resolved manner using PIV and PLIF. The following are the conclusions from this work.

1. The puffing frequency is independent of plume exit conditions since the instability is buoyancy driven. St calculated in the present case is in agreement with the $S t-R i_{\infty}$ scaling proposed by Cetegen \& Kasper (1996) for plumes emanating from nozzles.

2. St for pulsating plumes depends weakly on Re suggesting the mechanism for puffing in circular plumes to be inviscid, similar to that observed by Soteriou et al (2002) in planar plumes.

3. The flow field reveals that the plume entrains ambient fluid at all times during a puffing cycle. Velocity measurements reveal that the formation, convection and breakdown of the vortex ring formed in a puffing cycle is the primary mechanism for the entrainment in the near-field of the plumes. Density measurements reveal that centerline density remains preserved without dilution up to larger axial distances. The plume fluid in the near field is not diluted except at the thin interface, implying negligible effect of mass diffusion.

4. The relative contribution of unsteady and out flux terms in mass conservation equation to mass entrainment rate is investigated. Though the flow is unsteady, the contribution of unsteady 
mass term to the mass entrainment rate is negligible and is zero over a puff cycle. It can be concluded that a mean flow field is sufficient to characterize mass entrainment rate in pulsating plumes.

5. From the present experiments, a correlation has been proposed for the variation of mass entrainment rate along plume height (along axis) for pulsating buoyant plumes emanating from orifices. The correlation is found to follow a power law as shown below.

$$
\frac{\dot{m}_{\text {ent }}(Z)}{\dot{m}_{\text {in }}}=0.028\left(\operatorname{Re}_{\infty} i_{\infty} \frac{Z}{D}\right)^{0.73}
$$

\section{Acknowledgements}

The authors would like to thank Bhabha Atomic Research Center (BARC) and IITK PG Research Grant 2012 for their financial support.

\section{References}

Cetegen B M and Ahmed T A 1993 Experiments on the periodic instability of buoyant plumes and pool fires. Combust. Flame 93: 157-184

Cetegen B M and Kasper K D 1996 Experiments on the oscillatory behaviour of buoyant plumes of helium and helium-air mixtures. Phys. Fluids 8: 2974-2984

Cetegen B M 1997a Behavior of naturally unstable and periodically forced axisymmetric buoyant plumes of helium and helium-air mixtures. Phys. Fluids 9: 3742

Cetegen B M 1997b Measurements of instantaneous velocity field of a non-reacting pulsating buoyant plume by particle image velocimetry. Combust. Sci. Tech. 123: 377-387

Cetegen B M, Dong Y and Soteriou M C 1998 Experiments on stability and oscillatory behavior of planar buoyant plumes. Phys. Fluids 10: 1658

Clemens N T 2002 Flow imaging: Encyclopedia of imaging science and technology. New York: Wiley

DesJardin P E, O'Hern T J and Tieszen S R 2004 Large eddy simulation and experimental measurements of the near-field of a large turbulent helium plume. Phys. Fluids 16: 1866-1883

Jermy M C 2002 An economical droplet fog generator suitable for laser Doppler anemometry and particle imaging velocity seeding. Exp. Fluids 33: 321-322

Jiang X and Luo K H 2000 Direct numerical simulation of the puffing phenomenon of an axisymmetric thermal plume. Theoret. Comput. Fluid Dynamics 14: 55-74

Lozano A, Yip B and Hanson R K 1992 Acetone: A tracer for concentration measurements in gaseous flows by planar laser-induced fluorescence. Exp. Fluids 13: 369-376

Maragkos G, Rauwoens P, Wang Y and Merci B 2013 Large eddy simulations of the flow in the near-field region of a turbulent buoyant helium plume. Flow Turbulence Combust. 90: 511-543

Martin J E and Garcia M H 2009 Combined PIV/PLIF measurements of a steady density current front. Exp. Fluids 46: 265-276

O'Hern T J, Weckman E J, Gerhart A L, Tieszen S R and Schefer R W 2005 Experimental study of a turbulent buoyant helium plume. J. Fluid Mech. 544: 143-171

Soteriou M C, Dong Y and Cetegen B M 2002 Lagrangian simulation of unsteady near-field dynamics of planar buoyant plumes. Phys. Fluids 14: 3118-3140

Thurber M C 1999 Acetone laser-induced fluorescence for temperature and multi-parameter imaging in gaseous flows. Topical Report TSD-120, Thermo sciences Division, Department of Mechanical Engineering, Stanford University

Yildirim B S and Agrawal A K 2005 Full-field measurements of self-excited oscillations in momentumdominated helium jets. Exp. Fluids 38: 161-173 